\begin{tabular}{|c|l|}
\hline Title & Neuromagnetic responses to chords are modified by preceding musical scale \\
\hline Author(s) & Otsuka, A suka; Kuriki, Shinya; Murata, Noboru; Hasegawa, Toshikazu \\
\hline Citation & $\begin{array}{l}\text { Neuroscience Research, 60(1), 50-55 } \\
\text { https://doi.org/10.1016/.neures.2007.09.006 }\end{array}$ \\
\hline Issue Date & 2008 -01 \\
\hline Doc URL & http://hdl.handle.net/2115/32657 \\
\hline Type & article(author version) \\
\hline File Information & NR60-1.pdf \\
\hline
\end{tabular}

Instructions for use 


\section{Neuromagnetic responses to chords are modified by preceding musical scale}

Asuka Otsuka $^{\mathrm{a}}$, Shinya Kuriki ${ }^{\mathrm{b}}$, Noboru Murata ${ }^{\mathrm{c}}$ and Toshikazu Hasegawa ${ }^{\mathrm{a}}$

${ }^{a}$ Center for Evolutionary Cognitive Sciences, The University of Tokyo, Tokyo, Japan

${ }^{\mathrm{b}}$ Research Institute for Electronic Science, Hokkaido University, Sapporo, Japan

${ }^{\mathrm{c}}$ Department of Electrical Engineering and Bioscience, Waseda University, Tokyo, Japan

*Corresponding author

Asuka Otsuka

Tel : +81-3-5454-6709

Fax: +81-3-5454-6709

Email: otsuka@ecs.c.u-tokyo.ac.jp

Center for Evolutionary Cognitive Sciences, University of Tokyo, 3-8-1 Komaba, Meguro-ku, Tokyo 153-8902, Japan

Total number of pages: 22

Number of figures: 4

Number of tables: $\quad 0$ 


\title{
Neuromagnetic responses to chords are modified by preceding musical scale
}

\begin{abstract}
Previous psychological studies have shown that musical chords primed by Western musical scale in a tonal and modal schema are perceived in a hierarchy of stability. We investigated such priming effects on auditory magnetic responses to tonic-major and submediant-minor chords preceded by major scales and tonic-minor and submediant-major chords preceded by minor scales. Musically trained subjects participated in the experiment. During MEG recordings, subjects judged perceptual stability of the chords. The tonic chords were judged to be stable, whereas the submediant chords were judged to be unstable. Dipole moments of N1m response originating in the auditory cortex were larger in the left hemisphere for the submediant chords than for the tonic chords preceded by the major but not minor scales. No difference in the N1m or P2m moment was found for the chords presented without preceding scales. These results suggest priming effects of the tonal schema, interacting with contextual modality, on neural activity of the auditory cortex as well as perceptual stability of the chords. It is inferred that modulation of the auditory cortical activity is associated with attention induced by tonal instability and modality shift, which characterize the submediant chords.
\end{abstract}

Keywords: MEG, neuromagnetic response, auditory evoked field, chord, tonality, modality 


\section{Introduction}

The structural framework of Western tonal music is generally determined by a tonal and modal schema, such as "C major", representing the key or a note defining the tonal center (C) and modality (major). Modality of either major or minor is decided by intervallic arrangements of notes within an octave. Major chords are formed by notes having intervals of 4 and 7 semitones, whereas the intervals in minor chords are 3 and 7 semitones. Such a difference in modality is known to induce particular emotional effects in the listeners' cognition even when the chords are presented singly (Pallesen et al., 2003). On the other hand, tonality is assigned to tones and chords when they are referenced to a particular note. The diatonic tones and chords, denoted as I, II, to VII in order of ascending pitch, are perceived in a hierarchy of stability (Krumhansl, 2004), serving for the basis for the concept of tonality. The tonal hierarchy plays an important role in the perception of structural dynamism such as progression and closure of Western tonal music.

Krumhansl, with her colleagues, performed behavioral experiments to evaluate the tonal hierarchy of chords quantitatively using listeners' ratings for perceptual stability (Krumhansl \& Shepard, 1979; Krumhansl \& Kessler, 1982; Krumhansl, 1990). Their experimental paradigm is called the "probe-tone method", which has been widely used in the field of music psychology (Cuddy \& Badertcher, 1987; Steinke et al., 1997; Leman, 2000). In this method, subjects judge the stability of chords that have been primed tonally and modally by preceding musical context such as diatonic scales. The results showed that the perceptual stability was highest for tonic chords (I), e.g., CEG in 
C major context, which was followed by dominant (V) and subdominant chords (IV), and much lower for submediant chords (VI) , e.g., ACE. Similar patterns of hierarchical ordering have been observed for chords primed in major and minor contexts (Krumhansl, 1990). Interestingly, however, the chords in major context obtained finer discriminations, resulting in an increased difference between the tonic (I) and submediant (VI) chords in major than minor context. These results suggest an interactive effect of the tonal and modal schema on perception of chords primed by musical context.

The auditory perceptual/cognitive process has been widely investigated in relation to N1m and P2m responses of the auditory evoked fields (AEF). The AEFs reflect exogenous factors of input sounds (Pantev et al., 1989; Hari et al., 1990; Mizouchi et al., 2005; Tiitinen et al., 2005; Kuriki et al., 2006, 2007). They are also modulated by selective attention (Hari et al., 1989; Woldorff et al., 1993; Petkov et al., 2004; Neelon et al., 2006a, 2006b), i.e., a top-down modulation of the auditory cortical activity caused by an instruction or self-initiated decision to attend to the signal (Frith et al., 1996). Deviation from musical context such as melody and harmonic progression enhances the activity in the auditory cortex over the N1m and P2m latencies (Kuriki et al., 2005) and that in the inferior frontolateral cortex in/after the P2m latency (Maess et al., 2001). The enhancement of these cortical activities may be associated with selective attention. Selective attention prerequisites, by detection of contextual deviance, comparison and separation of an input sound (Giard et al., 2000), i.e., a process of template matching of an input sound with a template which is formed according to the task or the rule or pattern of preceding sounds. The results of Krumhansl's probe-tone experiment 
(Krumahnsl, 1990) behaviorally suggest that the tonic, i.e., the tonal center, functions as the template and that the precision of template matching depends on the modality of the priming context. However, there has been no studies on such a priming effect of tonality interacting with contextual modality on musical chords in relation to the modulation of auditory cortical activity as indexed by AEFs.

In this study, we investigated the auditory neuromagnetic responses, N1m and P2m, elicited by tonic (I) and submediant (VI) chords primed in major and minor contexts. The probe-tone method (Krumhansl, 1990) was adopted as the experimental paradigm. As a control, the same set of the chords were presented without any priming contexts. We hypothesized that auditory responses to the chords are modulated as an interactive function of the tonal and modal schema that primes the chords. Specifically, the response to the submediant chord (VI) in major context, which showed the lowest perceptual stability in Krumhansl's data, would be augmented by an attentional effect compared with that to the tonic chord (I) in major context, as primed by the preceding context.

\section{Methods}

Nine right-handed subjects (4 males and 5 females) of 20 to 26 years of age (mean age of 21.4 years) with normal hearing and no history of otological or neurological disorder participated in the experiment. They were all amateur string players of a university symphony orchestra who had been taking private musical lessons for one or 
more instruments since 3-16 (mean of 7.6) years of age continuously to the time of measurements. The experiment was approved by the Ethics Committee of Hokkaido University. The subjects gave informed consent in writing for participation in the study after the purpose and procedures of the experiment had been fully explained.

Figure 1 (top) shows an example of musical score of stimulus sounds composed of a diatonic scale made of 15 notes ascending in pitch in two octaves and a four-note chord. The chords of the sequences were characterized tonally and modally as tonic-major and submediant-minor chords preceded by a major scale, denoted as $\mathrm{I}_{\text {maj }}$ and $\mathrm{VI}_{\text {maj, }}$, respectively, and tonic-minor and submediant-major chords preceded by a minor scale, denoted as $\mathrm{I}_{\min }$ and $\mathrm{VI}_{\min }$, respectively. In order to offset the effect of a particular key, the sequences were prepared in two different keys (Fig. 1, middle), which resulted in tuning the chords for $\mathrm{I}_{\text {maj }}$ in C major with notes of C5E5G5C6 (denoted as C) and in F major with notes of F5A5C6F6 (F). The chords for $\mathrm{VI}_{\text {maj }}$ were tuned in A minor with notes of A4C5E5A5 $\left(\mathrm{A}_{\mathrm{m}}\right)$ and in D minor with D5F5A5D6 $\left(\mathrm{D}_{\mathrm{m}}\right)$. These chords of C/F and $A_{m} / D_{m}$ were preceded by $C$ major and F major scales, respectively. The chords for $I_{\text {min }}$ were tuned in E minor with notes of E4G4B4E5 $\left(E_{m}\right)$ and in A minor $\left(A_{m}\right)$, and the chords for $\mathrm{VI}_{\min }$ were tuned in $\mathrm{C}$ major $(\mathrm{C})$ and $\mathrm{F}$ major $(\mathrm{F})$. These chords of $\mathrm{E}_{\mathrm{m}} / \mathrm{A}_{\mathrm{m}}$ and C/F were preceded by E-natural minor and A-natural minor scales, respectively. It should be noted that the chords $\mathrm{C} / \mathrm{F}$ of $\mathrm{I}_{\text {maj }}$ were identical to the chords $\mathrm{C} / \mathrm{F}$ of $\mathrm{VI}_{\text {min }}$ except for the key and modality of the preceding scale.

The stimulus sounds were generated in standard piano timber in MIDI format (Yamaha, XGworks v.4.0) and converted to WAV format (Adobe Systems, Inc., Audition v.1.0). The duration of the chord was 312.5 ms with a 10-ms ramp of linear 
decrease applied at the end. The interval between the onset of the last note of the scale and the onset of the chord was $1250 \mathrm{~ms}$. We measured the hearing threshold for each chord in individual subjects in the same condition of binaural stimulation as in the MEG recording. The sound intensity was adjusted for each of five different chords, i.e., C, F, $A_{m}, D_{m}$ and $E_{m}$, to a level of $60 \mathrm{~dB}$ above the hearing threshold. The mean sound pressure level (SPL) of the five chords in each subject was 68.0 - $71.0 \mathrm{~dB}$, which was used to adjust the SPL of the notes of the preceding scale.

MEG measurements were made using a custom-made helmet-shaped SQUID system with 76-channel magnetometer sensors (Elekta-Neuromag, Helsinki). In the main session, the sequences composed of the scales and chords, $\mathrm{I}_{\mathrm{maj}}, \mathrm{VI}_{\mathrm{maj}}, \mathrm{I}_{\text {min }}$ and $\mathrm{VI}_{\text {min }}$, tuned in two different keys, were mixed in pseudo-random order and repeated for more than 50 times. The onset-to-onset interval of the sequences was 6250 ms. Subjects were instructed to listen to the stimulus sequence and judge whether the chord presented at the end was stable or unstable, with regard to the preceding scale. The subjects responded by moving the right or left index finger at a response time notified with a drum sound after the MEG recording period (Fig. 1, bottom). In the control session, all of the five chords, $C, F, A_{m}, D_{m}$ and $E_{m}$, were presented singly without a preceding scale and repetitively for more than 50 times. The onset-to-onset interval of the chords was 625 ms. Subjects were not required to perform any task other than listening passively to the single chords. Magnetic signals of $100 \mathrm{~ms}$ before and up to $500 \mathrm{~ms}$ after onset of the chord were recorded in both main and control sessions. Epochs contaminated with artifacts exceeding 3.0 pT were rejected online, resulting in removal of less than five epochs in single conditions. 
The recorded magnetic signals were bandpass-filtered offline using a fourth-order bidirectional Butterworth filter with a cutoff frequency of 1-20 Hz. This relatively narrow bandwidth was used to reduce the low-frequency and power line (50 Hz) noises in the environment, which existed at high amplitudes in the signals detected by the magnetometer-type sensors. A notch filter at $50 \mathrm{~Hz}$ (3.14 Hz width) was also applied to assure the removal of the power line noise. Magnetic signals of 50 epochs were averaged for each chord separately. Averages of 100 epochs were made by summing further the chords of $C$ and $F, A_{m}$ and $D_{m}$, and $E_{m}$ and $A_{m}$, respectively, for conditions of $I_{m a j}, V I_{m a j}, I_{\min }$ and $V I_{m i n}$ in the main session and $C / F, A_{m} / D_{m}$ and $E_{m} / A_{m}$ in the control session. The mean of the signal in a 100 -ms period before onset of the chord served as the baseline.

The equivalent current dipoles were localized separately for the N1m and P2m components elicited by each chord. The estimation was performed using 38-channel field data from each hemisphere of each subject. The coordinates and directions of N1m and P2m dipoles were not statistically different among the chords or between the sessions in each hemisphere, as examined by one-way repeated-measures ANOVA with a factor of chord or session. We therefore averaged all of the chords in both sessions, from which template dipoles having the mean coordinates and directions were obtained for N1m and P2m components in the left and right hemispheres. The moment was further normalized with its magnitude. The time course of dipole moment was calculated by the signal space projection (SSP) method (Tesche et al., 1995; Fujioka et al., 2005; Kuriki et al., 2006) by projecting the measured neuromagnetic fields on the fields that were given by the template dipoles for N1m and P2m components. This 
method provides a quantitative measure of the dipole moment even from data of low signal-to-noise ratio. The magnitudes of the projected moment for the N1m and P2m components were obtained at their peak latencies.

The projected moments of N1m and P2m components were assessed statistically by two-way repeated-measures ANOVA with factors of hemisphere and session and post-hoc Scheffe's tests. The analysis was performed for each pair of corresponding conditions, i.e., $\mathrm{C} / \mathrm{F}$ and $\mathrm{I}_{\mathrm{maj}}, \mathrm{A}_{\mathrm{m}} / \mathrm{D}_{\mathrm{m}}$ and $\mathrm{VI} \mathrm{I}_{\mathrm{maj}}, \mathrm{E}_{\mathrm{m}} / \mathrm{A}_{\mathrm{m}}$ and $\mathrm{I}_{\mathrm{min}}$, and $\mathrm{C} / \mathrm{F}$ and $\mathrm{VI} \mathrm{I}_{\mathrm{min}}$, across the control and main sessions. In order to examine the difference among the dipole moments of $I_{\text {maj }}, \mathrm{VI}_{\mathrm{maj}}, \mathrm{I}_{\mathrm{min}}$, and $\mathrm{VI}_{\min }$ in the main session, three-way repeated-measures ANOVA was performed with factors of hemisphere, modality of preceding scale (major or minor) and tonality of chord (I or VI). The conditions of C/F, $A_{m} / D_{m}, E_{m} / A_{m}$ in the control session were examined separately by two-way repeated-measures ANOVA with factors of hemisphere and chords.

\section{Results}

The behavioral judgments of stability of the chords were consistent among subjects.

The group mean across subjects showed that $\mathrm{I}_{\text {maj }}$ and $\mathrm{I}_{\min }$ were judged as stable in $96.5 \%$ (1.6\% of SE) and $85.3 \%$ (5.6\%) of 100 epochs, respectively, while $\mathrm{VI}_{\text {maj }}$ and $\mathrm{VI}_{\text {min }}$ were judged as unstable in 90.1\% (3.6\%) and 91.7\% (3.5\%) of epochs, respectively. These percent values were well above the chance level of judgment, being significantly larger than $50 \%(\mathrm{p}<0.001)$. 
Figure 2a shows the waveform of superposed fields of 38 channels over the right hemisphere of one subject for the response to the chord of $\mathrm{I}_{\text {maj. }}$ Clearly elicited N1m and P2m components of neuromagnetic response were observed at latencies of about 100 and $170 \mathrm{~ms}$, respectively, after onset of the chord. The dipolar pattern of the measured field distribution over the Sylvian fissure and also the mean location of the dipoles (Fig. 2a) in the structure of a template brain (Neuroscan, Curry 5.0.8) indicated a single current dipole source for each component in the auditory area of the superior temporal cortex. Figure 2b displays grand-mean $(n=9)$ coordinates of N1m and P2m dipoles for each chord in the control and main sessions. One-way repeated-measures ANOVA with a factor of chord or session showed no significant difference in the N1m and P2m dipole coordinates and orientations in each hemisphere. These results support the validity of using the mean locations among the chords and between the sessions as SSP templates for N1m and P2m components. Figure 2c shows the dipole moments obtained by projecting the measured fields to the template fields of the grand-mean single source of the N1m component, where the results of the grand-mean and different subjects are superposed. Such a dipole moment as a function of latency represents the time course of the source activity, when a single source of the N1m component is assumed. The group means of the source activity for different chords (Fig. 3) indicated a slight difference in the amplitude of the $\mathrm{N} 1 \mathrm{~m}$ moment for $\mathrm{VI}_{\mathrm{maj}}$ in the left hemisphere for the main session. The source activity for the P2m component calculated using the grand-mean template dipole of the P2m moment showed a similar result for $\mathrm{VI}_{\text {maj. }}$.

Quantitative evaluation of the N1m and P2m moments was made from their peak values of the source activities in different conditions (Fig. 4). The two-way 
repeated-measures ANOVA with factors of hemisphere and session showed the main effect of session between the pairs of $A_{m} / D_{m}$ and $V I_{m a j}\left(F_{(1,8)}=10.47 ; p<0.01\right), E_{m} / A_{m}$ and $\mathrm{I}_{\min }\left(\mathrm{F}_{(1,8)}=10.03 ; \mathrm{p}<0.01\right)$, and $\mathrm{C} / \mathrm{F}$ and $\mathrm{VI}_{\min }\left(\mathrm{F}_{(1,8)}=5.08 ; \mathrm{p}<0.05\right)$, with the $\mathrm{N} 1 \mathrm{~m}$ moments in the main session being significantly larger than those in the control session. There was also an interaction between session and hemisphere in the pair of $\mathrm{C} / \mathrm{F}$ and $\mathrm{I}_{\text {maj }}$ $\left(F_{(1,8)}=5.77 ; p<0.04\right)$, with the N1m moments to $I_{\text {maj }}$ in the right hemisphere being marginally larger than those in the left hemisphere in the main session $(\mathrm{p}<0.06)$. No significant effects were found in any pairs of the P2m moments. The repeated-measures ANOVA with factors of hemisphere, modality of preceding scale and tonality of chord showed a three-way interaction effect $\left(\mathrm{F}_{(1,8)}=6.73\right.$; $\left.\mathrm{p}<0.03\right)$ on the $\mathrm{N} 1 \mathrm{~m}$ moment for the main session. A post-hoc Scheffe's test revealed that $\mathrm{VI}_{\text {maj }}$ was significantly larger than $I_{\text {maj }}(p<0.05)$ in the left hemisphere. No significant differences were found in the right hemisphere. No effects of any factors were observed for the P2m moments. Also, the results for the control session showed no significant effects on the N1m and P2m moments.

\section{Discussion}

In the behavioral performance, tonic chords $\mathrm{I}_{\text {maj }}$ and $\mathrm{I}_{\min }$ were judged to be stable and submediant chords $\mathrm{VI}_{\text {maj }}$ and $\mathrm{VI}_{\text {min }}$ were judged to be unstable. The results agree with the behavioral data reported by Krumhansl (1990). Specifically, the different judgment obtained for the acoustically identical chords, $\mathrm{I}_{\text {maj }}$ and $\mathrm{VI}_{\min }$, suggests that the priming 
of the preceding musical scale functioned to induce hierarchy in the perceptual stability of the chords, i.e., tonality.

There was a significant interaction effect of tonality by contextual modality in the main session, with $\mathrm{VI}_{\text {maj }}$ eliciting significantly larger $\mathrm{N} 1 \mathrm{~m}$ moments than $\mathrm{I}_{\text {maj }}$ in the left hemisphere. This tonal-modal interaction effect confirmed our hypothesis that the factors of tonality of the chords and contextual modality defined by the preceding scale are reflected interactively in the auditory cortical activities. The significant difference between $\mathrm{VI}_{\text {maj }}$ and $\mathrm{I}_{\text {maj }}$ agrees with the maximum difference in the ratings of perceptual stability between I and VI in major modality (Krumhansl, 1990), suggesting the involvement of cortical activity at the N1m latency in the subsequent cognitive judgment. The chords presented without the preceding scales in the control session, $\mathrm{C} / \mathrm{F}$ and $A_{m} / D_{m}$, which acoustically corresponded to $I_{\text {maj }}$ and $V I_{\text {maj }}$ of the main session, showed no difference in amplitude, in agreement with the results of a previous fMRI study (Pallesen et al., 2005). This supports the notion that the difference between the $\mathrm{N} 1 \mathrm{~m}$ responses to $\mathrm{I}_{\text {maj }}$ and $\mathrm{VI}_{\text {maj }}$ was a result of priming by the preceding context and was not caused by the acoustic features of the chords.

Kuriki et al. (2004) recorded neuromagnetic responses elicited by final notes tuned in I or IV\#, e.g., C or F\# in C major context, of musical melodies and observed that the auditory cortical activities were significantly larger to IV\# than to I over the latencies of N1m and P2m responses. In Western diatonic music composed in the tonal-modal schema, strong expectancy for the tonal center, I, is induced at the end of the musical progression (Tillman et al., 2003). In contrast, IV\# deviates from the anticipated tonality (I) and also violates the tonal-modal rule established by the preceding part of the 
melodies. Similarly, in the present study, $\mathrm{VI}_{\text {maj }}$ was a deviation in terms of modality, i.e., a minor chord in major context, and also tonality, i.e., a submediant chord that is distant from the tonal center defined by the preceding scale. Assuming that the chords primed in the tonal-modal schema were processed in reference to the tonic chord, $\mathrm{I}_{\text {maj }}$, that functioned as a template, it would be efficient for the subjects to perform the task by initiating their attention to the deviant chord, $\mathrm{VI}_{\mathrm{maj}}$, that did not fit with the template. Then the selective attention may induce augmentation of the $\mathrm{N} 1 \mathrm{~m}$ activity for $\mathrm{VI}_{\text {maj. }}$. We speculate that such cortical modulation could occur at an earlier stage in parallel with acoustic analysis of input sound in the auditory cortex, through the efferent influence of attention on the expeditious filtering of irrelevant/unattended (i.e., template) sounds at peripherals (Giard et al., 1994; 2000). It should be noted that $\mathrm{VI}_{\text {maj }}$ was not a physical oddball in a regular pattern. Furthermore, $\mathrm{VI}_{\text {maj }}$ was presented at an equal frequency with the other conditions for $\mathrm{I}_{\mathrm{maj}}, \mathrm{I}_{\min }$ and $\mathrm{VI}_{\min }$. Therefore, the mechanism underlying the augmentation of $\mathrm{N} 1 \mathrm{~m}$ activity for $\mathrm{VI}_{\text {maj }}$ may be distinct from sensory adaptation and gating effect of the feature-specific auditory neurons (Hari et al., 1982), which is partly associated with the classical mismatch negativity (MMN) generation mechanism (Jaaskelainen et al., 2004).

In contrast, $\mathrm{VI}_{\min }$ did not augment the responses in spite of the common characteristics of tonality (VI), perceptual stability and modality shift from the preceding scale. Krumhansl (1990) reported a difference in perceptual stability as a function of contextual modality, with the chords in major context obtaining finer discriminations than those in minor context. A previous psychological study also showed that major context predisposes musically trained subjects to apprehend auditory 
regularity (Oram and Cuddy, 1995). It is therefore suggested that augmentation of the response to $\mathrm{VI}_{\mathrm{maj}}$, but not to $\mathrm{VI}_{\min }$, is associated with the subjects' sensitivity to the tonal schema established more firmly by the preceding scales of the major modality.

The notes contained in $I_{\text {maj }}$ were presented near the end of the preceding scale, whereas the notes in $\mathrm{I}_{\min }$ occurred in the first half of the scale. The notes in $\mathrm{VI}_{\text {maj }}$ and $\mathrm{VI}_{\min }$ occurred in the middle part of the scale. Thus, the effect of adaptation due to frequency overlap is expected to be maximal for $\mathrm{I}_{\text {maj, }}$, which may have contributed to the difference between $\mathrm{I}_{\text {maj }}$ and $\mathrm{VI}_{\text {maj. }}$.

A significant effect of chord was observed in the left, but not right, hemisphere. It has been reported that auditory responses are enhanced in musically trained listeners bilaterally with right hemisphere dominance (Shahin, et al., 2003; Kuriki, et al., 2004; Bosnyak, et al., 2004). However, piano tones elicit larger N1m response than envelope-matched pure tones do in the left hemisphere of both musicians and non-musicians (Lütkenhöner, et al., 2006). Furthermore, the left hemisphere is known to show dominance in the analysis of pitch interval (Liégeois-Chauvel et al., 1998; Ehrlé et al., 2001; Samson et al., 2001). It is therefore suggested that the significant difference between the chords in the left hemisphere was a result of identification and differentiation of the frequency characteristics of the chords of piano timber, relative to the tonal center, I, determined by the preceding scale.

$\mathrm{N} 1 \mathrm{~m} / \mathrm{P} 2 \mathrm{~m}$ responses are attenuated by repetitive presentation of sounds due to the adaptation of auditory cortical neurons (Kuriki et al., 2007), which depends on the interval between the stimulus (Hari et al., 1982; Ioannides et al., 2003) and the frequency characteristics of preceding sounds (Watanabe, 2003). The observed effect of 
session on the N1m moments is thought to be mainly caused by the adaptation that existed strongly in the control session, due to the frequency overlapping by repetition of an identical chord and the short inter-stimulus-interval of $625 \mathrm{~ms}$. In the main session, intermission between the chord and the preceding tone was $1250 \mathrm{~ms}$, and only some of the frequency components were overlapped between the chord and the last note of the preceding scale.

\section{Acknowledgements}

The authors wish to thank D. Fukuda and K. Watanabe of Waseda University for their help in the experiment. 


\section{References}

Bosnyak, DJ., Eaton, RA., Roberts, LE., 2004. Distributed auditory cortical representations are modified when non-musicians are trained at pitch discrimination with $40 \mathrm{~Hz}$ amplitude modulated tones. Cereb Cortex. 14, 1088-1099.

Cuddy, LL., Badertscher, B., 1987. Recovery of the tonal hierarchy: some comparisons across age and levels of musical experience. Percept Psychophys. 41, 609-620.

Ehrlé, N., Samson, S., Baulac, M., 2001. Processing of rapid auditory information in epileptic patients with left temporal lobe damage. Neuropsychologia. 39, 525-531.

Frith, CD., Friston, KJ., 1996. The role of the thalamus in "top down" modulation of attention to sound. Neuroimage. 4, 210-215.

Fujioka, T., Trainor, LJ., Ross, B., Kakigi, R., Pantev, C., 2005. Automatic encoding of polyphonic melodies in musicians and nonmusicians. J Cogn Neurosci. 17, 1578-1592.

Giard, MH., Collet, L., Bouchet, P., Pernier, J., 1994. Auditory selective attention in the human cochlea. Brain Res. 633, 353-356.

Giard, MH., Fort, A., Mouchetant-Rostaing, Y., Pernier, J., 2000. Neurophysiological mechanisms of auditory selective attention in humans. Front Biosci. 5, 84-94.

Hari, R., Kaila, K., Katila, T., Tuomisto, T., Varpula, T., 1982. Interstimulus interval dependence of the auditory vertex response and its magnetic counterpart: implications for their neural generation. Electroencephalogr Clin Neurophysiol. 54, 561-569.

Hari, R., Hamalainen, M., Kaukoranta, E., Makela, J., Joutsiniemi, SL., Tiihonen, J., 1989. Selective listening modifies activity of the human auditory cortex. Exp Brain 
Res. 74, 463-470.

Hari, R., 1990. Magnetic evoked fields of the human brain: basic principles and applications. Electroencephalogr Clin Neurophysiol Suppl. 41, 3-12.

Ioannides, AA., Popescu, M., Otsuka, A., Bezerianos, A., Liu, L., 2003.

Magnetoencephalographic evidence of the interhemispheric asymmetry in echoic memory lifetime and its dependence on handedness and gender. Neuroimage. 19, 1061-1075.

Jaaskelainen, IP., Ahveninen, J., Bonmassar, G., Dale, AM., Ilmoniemi, RJ., Levanen, S., Lin, FH., May, P., Melcher, J., Stufflebeam, S., Tiitinen, H., Belliveau, JW., 2004. Human posterior auditory cortex gates novel sounds to consciousness. Proc Natl Acad Sci U S A. 101, 6809-6814.

Krumhansl, CL., Shepard, RN., 1979. Quantification of the hierarchy of tonal functions within a diatonic context. J Exp Psychol Hum Percept Perform. 5, 579-594.

Krumhansl, CL., Kessler, EJ., 1982. Tracing the dynamic changes in perceived tonal organization in a spatial representation of musical keys. Psychol Rev. 89, 334-368. Krumhansl, CL., 1990. Cognitive Foundations of Musical Pitch, Oxford Psychological Series, no. 17. Oxford University Press, New York.

Krumhansl, CL., 2004. The cognition of tonality - as we know it today. J New Music Res. 33, 253-268

Kuriki, S., Isahai, N., Otsuka, A., 2005. Spatiotemporal characteristics of the neural activities processing consonant/dissonant tones in melody. Exp Brain Res. 162, 46-55.

Kuriki, S., Kanda, S., Hirata, Y., 2006. Effects of musical experience on different 
components of MEG responses elicited by sequential piano-tones and chords. $\mathrm{J}$ Neurosci. 26, 4046-4053.

Kuriki, S., Ohta, K., Koyama, S., 2007. Persistent Responsiveness of Long-Latency Auditory Cortical Activities in Response to Repeated Stimuli of Musical Timbre and Vowel Sounds. Cereb Cortex. Epub ahead of print.

Leman, M., 2000. An Auditory Model of the Role of Short-Term Memory in Probe-Tone Ratings. Music Perception. 17, 481-509.

Liégeois-Chauvel, C., Peretz, I., Babai, M., Laguitton, V., Chauvel, P., 1998. Contribution of different cortical areas in the temporal lobes to music processing. Brain. 121, 1853-1867.

Lütkenhöner, B., Seither-Preisler, A., Seither, S., 2006. Piano tones evoke stronger magnetic fields than pure tones or noise, both in musicians and non-musicians. Neuroimage. 30, 927-937.

Maess, B., Koelsch, S., Gunter, TC., Friederici, AD., 2001. Musical syntax is processed in Broca's area: an MEG study. Nat Neurosci. 4, 540-545.

Mizuochi, T., Yumoto, M., Karino, S., Itoh, K., Yamakawa, K., Kaga, K., 2005.

Perceptual categorization of sound spectral envelopes reflected in auditory-evoked N1m. Neuroreport. 16, 555-558.

Neelon, MF., Williams, J., Garell, PC., 2006a. The effects of attentional load on auditory ERPs recorded from human cortex. Brain Res. 1118, 94-105.

Neelon, MF., Williams, J., Garell, PC., 2006b. The effects of auditory attention measured from human electrocorticograms. Clin Neurophysiol. 117, 504-521.

Oram, N., Cuddy, LL., 1995. Responsiveness of Western adults to pitch-distributional 
information in melodic sequences. Psychol Res. 57, 103-118.

Pallesen, KJ., Brattico, E., Carlson, S., 2003. Emotional connotations of major and minor musical chords in musically untrained listeners. Brain Cogn. 51, 188-190.

Pallesen, KJ., Brattico, E., Bailey, C., Korvenoja, A., Koivisto, J., Gjedde, A., Carlson, S., 2005. Emotion processing of major, minor, and dissonant chords: a functional magnetic resonance imaging study. Ann N Y Acad Sci. 1060, 450-453.

Pantev, C., Hoke, M., Lutkenhoner, B., Lehnertz, K., 1989. Tonotopic organization of the auditory cortex: pitch versus frequency representation. Science. 246, 486-488.

Petkov, CI., Kang, X., Alho, K., Bertrand, O., Yund, EW., Woods, DL., 2004. Attentional modulation of human auditory cortex. Nat Neurosci.7, 658-663.

Samson, S., 2001. Cerebral substrates for musical temporal processes. In The Cognitive Neuroscience of Music. In: Peretz, I., and Zatorre, RJ. (Eds.), The Cognitive Neuroscience of Music. Oxford University Press, New York, pp. 204-216.

Shahin, A., Bosnyak, DJ., Trainor, LJ., Roberts, LE., 2003. Enhancement of neuroplastic P2 and N1c auditory evoked potentials in musicians. J Neurosci. 23, 5545-5552.

Steinke, WR., Cuddy, LL., Holden, RR., 1997. Dissociation of musical tonality and pitch memory from nonmusical cognitive abilities. Can J Exp Psychol. 51, 316-334. Tesche, CD., Uusitalo, MA., Ilmoniemi, RJ., Huotilainen, M., Kajola, M., Salonen, O., 1995. Signal-space projections of MEG data characterize both distributed and well-localized neuronal sources. Electroencephalogr Clin Neurophysiol. 95, 189-200.

Tillmann, B., Janata, P., Bharucha, JJ., 2003. Activation of the inferior frontal cortex in 
musical priming. Brain Res Cogn Brain Res. 16, 145-161.

Tiitinen, H., Makela, AM., Makinen, V., May, PJ., Alku, P., 2005. Disentangling the effects of phonation and articulation: hemispheric asymmetries in the auditory N1m response of the human brain. BMC Neurosci. 6:62.

Watanabe, M., 2003. A neuromagnetic study on the characteristics of human auditory system: an investigation of the underlying mechanism for multiple tone processing. Doctoral dissertation. Faculty of Science and Engineering, Waseda University, Japan. http://hdl.handle.net/2065/409

Woldorff, MG., Gallen, CC., Hampson, SA., Hillyard, SA., Pantev, C., Sobel, D., Bloom, FE., 1993. Modulation of early sensory processing in human auditory cortex during auditory selective attention. Proc Natl Acad Sci U S A. 90, 8722-8726. 
Figure captions

Figure 1. Stimulus sequences and MEG recording. Musical score of stimulus sounds forming a sequence by combining a preceding scale and a target chord (top). The five chords, $C, A_{m}, F, D_{m}$ and $E_{m}$, primed by the scales in keys of either $C, F, E_{m}$ or $A_{m}$. The chords were assigned tonality of either I or VI to form four conditions of $\mathrm{I}_{\text {maj }}, \mathrm{VI}_{\text {maj }}, \mathrm{I}_{\text {min }}$ and $\mathrm{VI}_{\min }$ (middle). The time schematics of the stimulus sequence presentation, MEG recording and behavioural response are shown at the bottom.

Figure 2. Measured magnetic fields and calculated dipole moments of auditory evoked responses elicited by the chord of $\mathrm{I}_{\text {maj. }}$ (a) Superposed magnetic fields of 38 channels over the right hemisphere of one subject, with the field distributions and source locations and directions of equivalent current dipoles estimated at N1m and P2m peaks. (b) Grand-mean ( $\mathrm{n}=9$ ) coordinates of N1m and P2m dipoles projected on coronal and axial planes for each chord in the control and main sessions. (c) Dipole moments as a function of latency obtained by projecting the measured fields to the template fields of the grand-mean single source of N1m response. The results for each of nine subjects and their mean are indicated by thin and thick lines, respectively.

Figure 3. Grand-mean projected moments of N1m sources in the left (LH) and right (RH) hemispheres obtained in control and main sessions. The results of different conditions are compared by superposition.

Figure 4. Summarized results of the projected moments at (a) N1m and (b) P2m peaks in the left ( $\mathrm{LH})$ and right (RH) hemispheres obtained in control and main sessions. Significant difference and tendency between the conditions are indicated. Significant 
differences for the factors of session and hemisphere are omitted for clarity. 

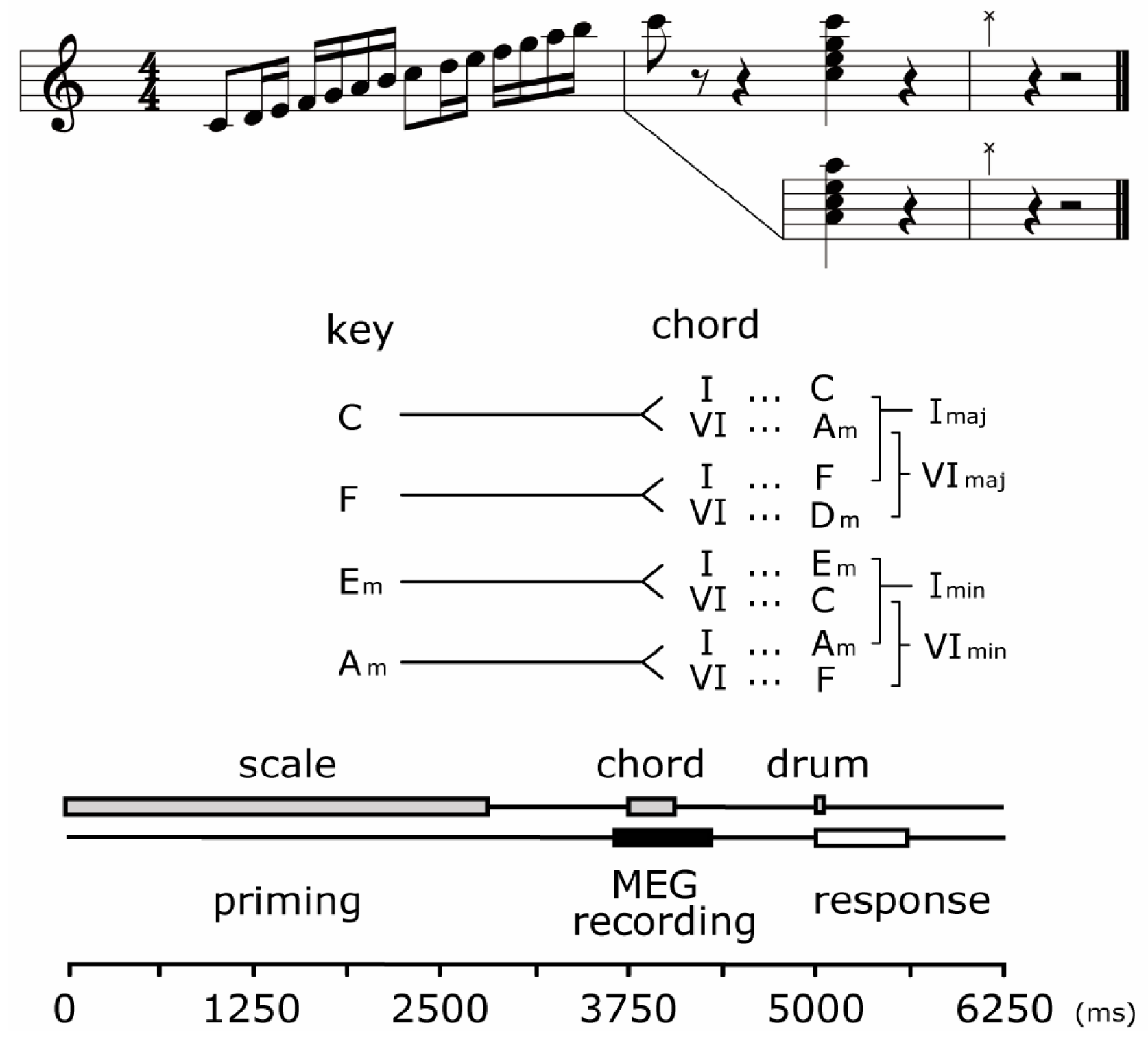

Figure 1. Otsuka et al.

Neuromagnetic responses to chords are modified by preceding musical scale 


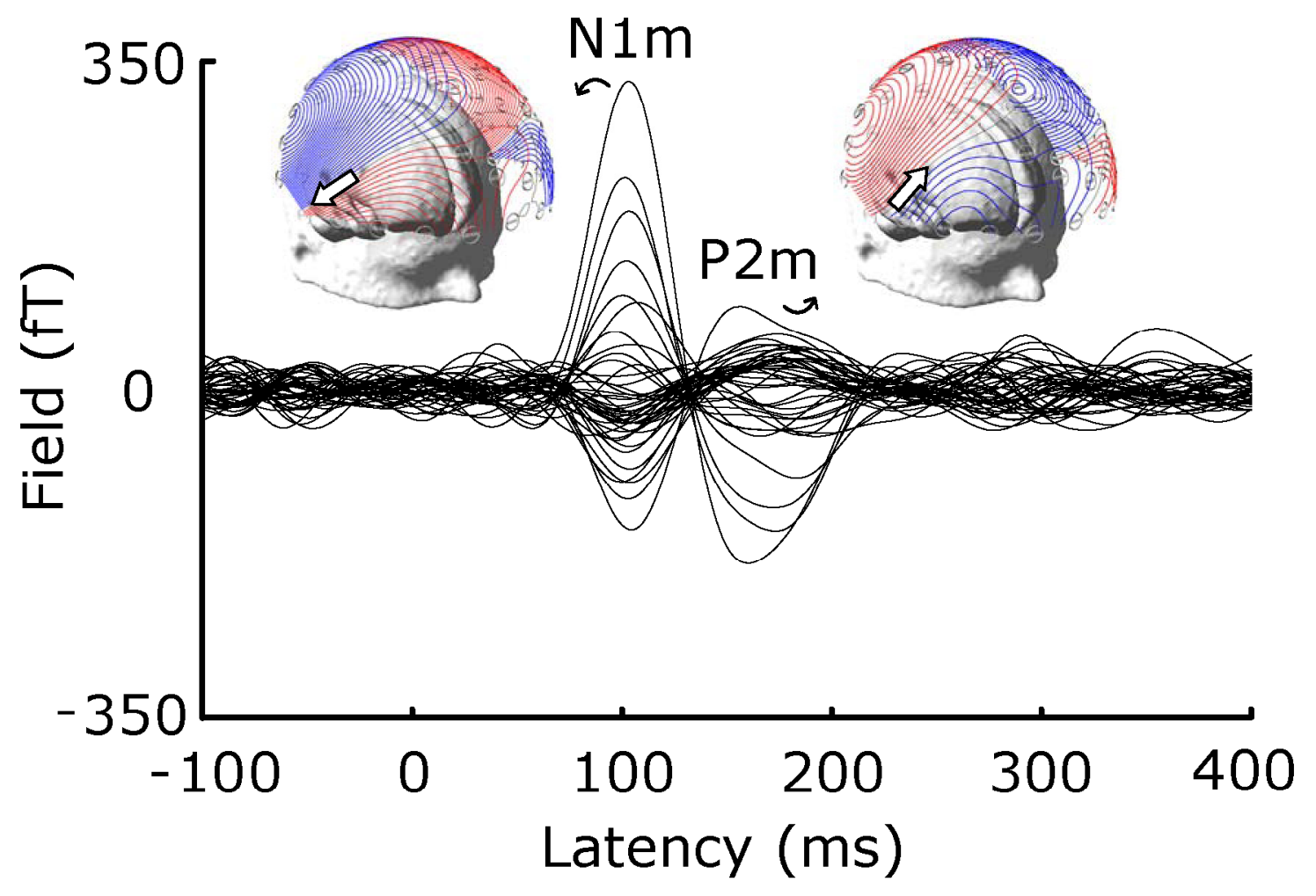

(a)

N1m location

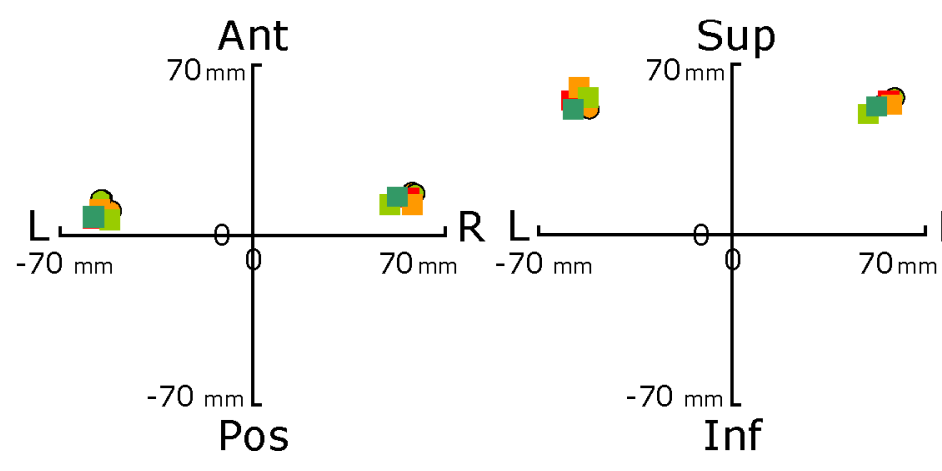

axial view coronal view
P2m location

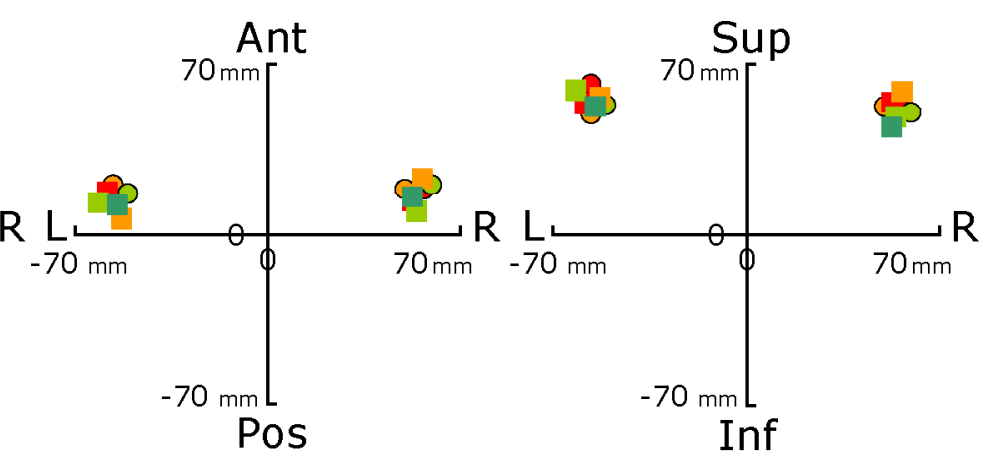

axial view coronal view control main
- $\mathrm{C} / \mathrm{F}$
- $A m / D m$
- $E m / A m$
- Imaj
- VImaj
- Imin
- VImin

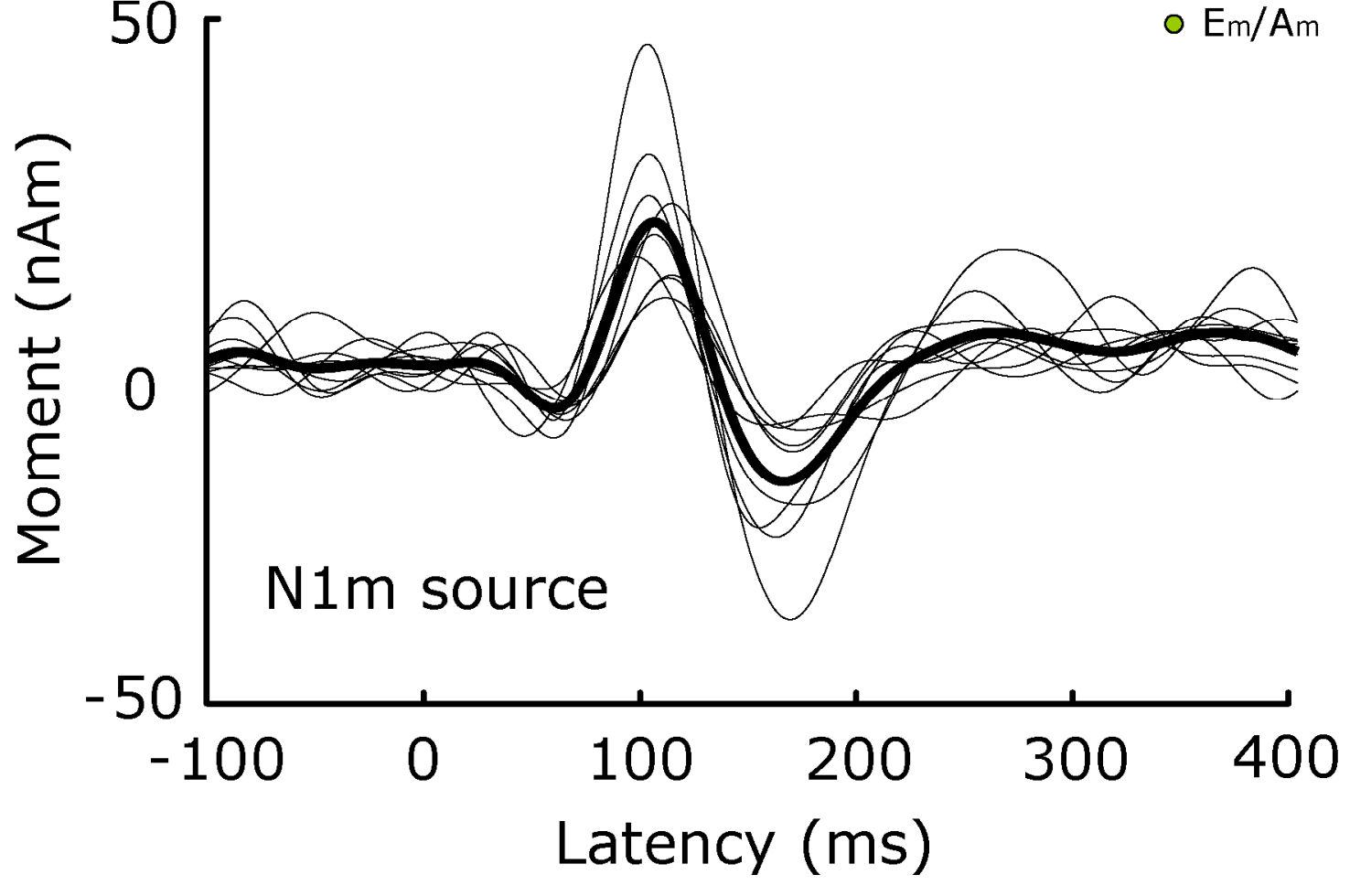




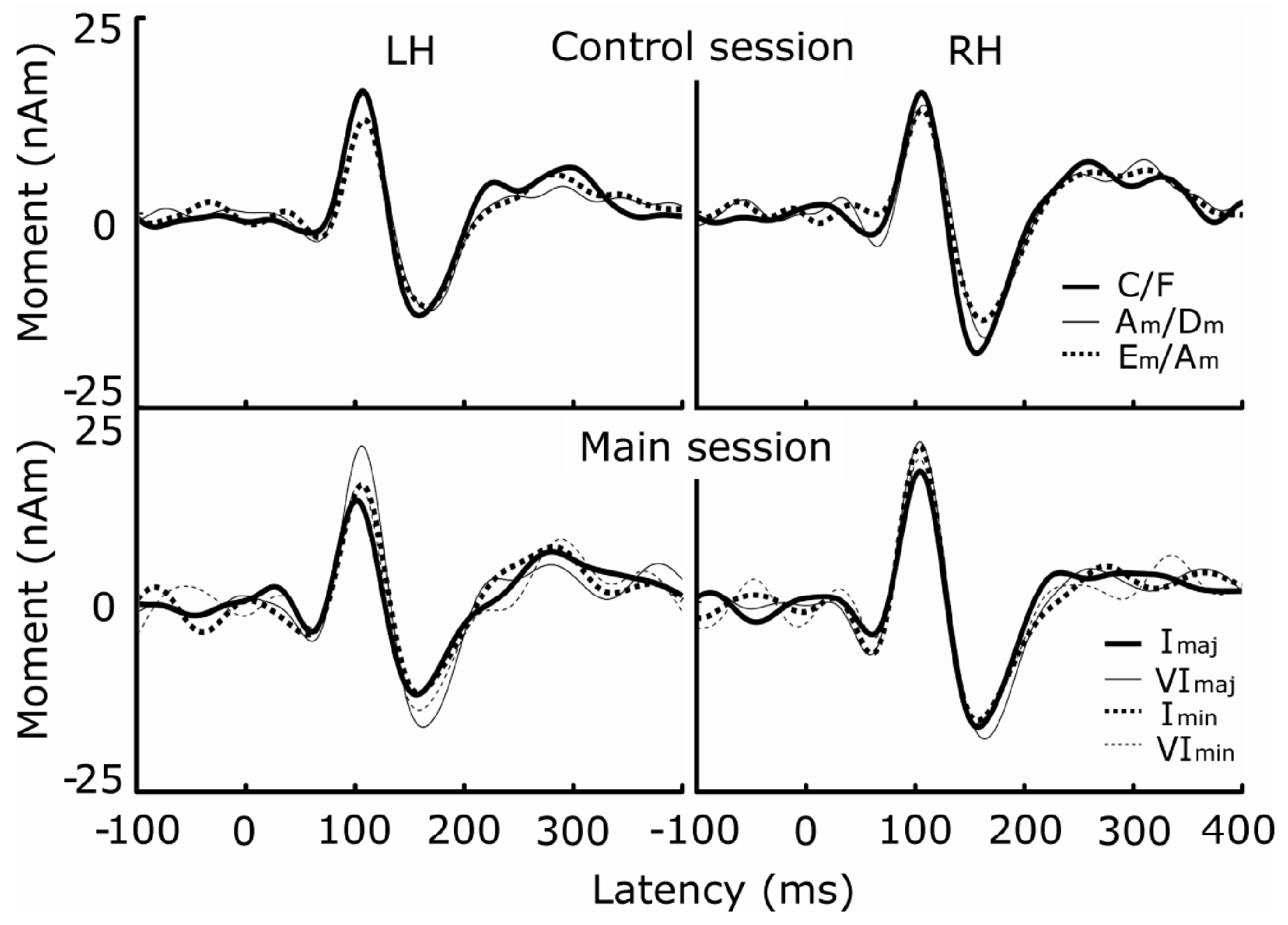

Figure 3. Otsuka et al.

Neuromagnetic responses to chords are modified by preceding musical scale 


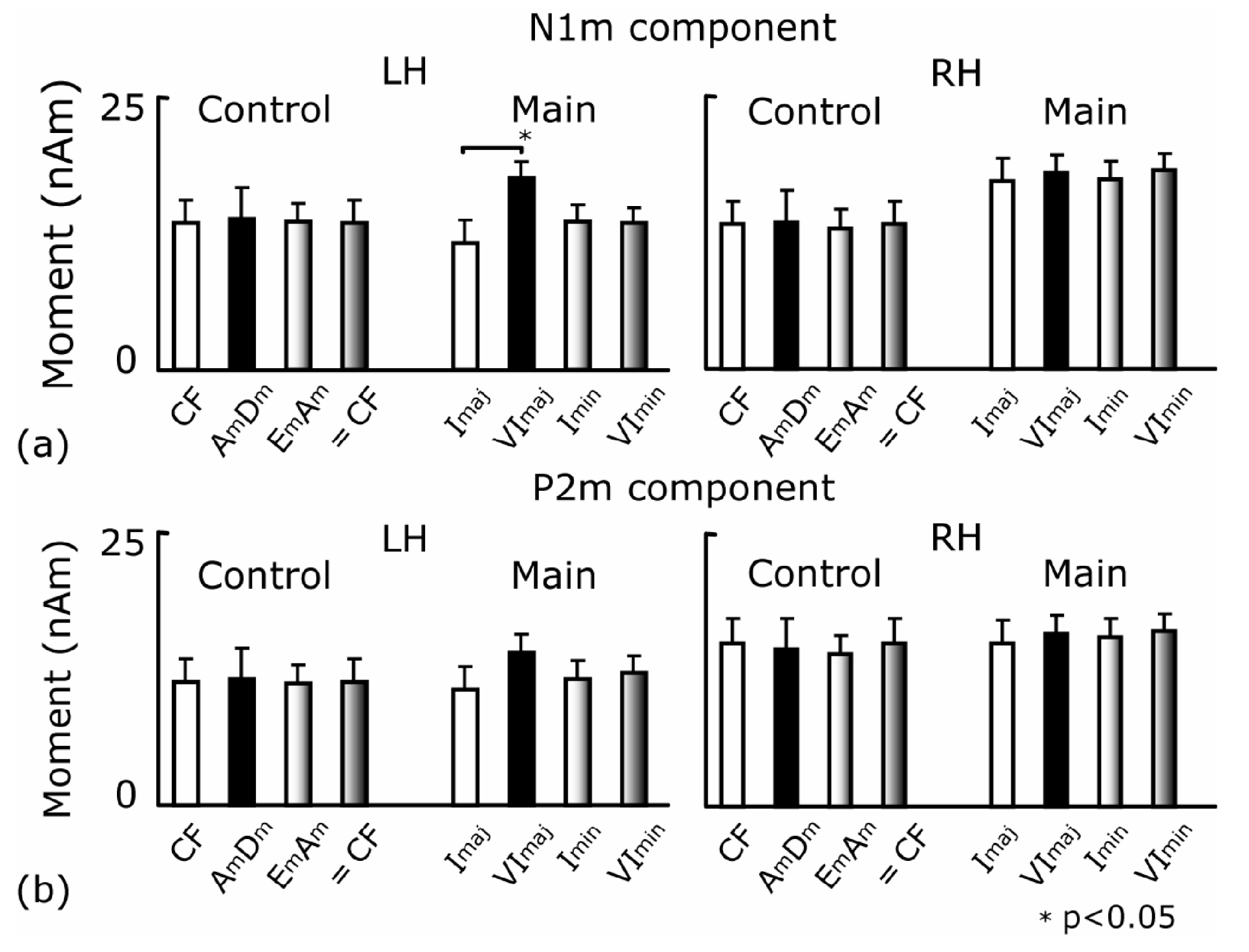

Figure 4. Otsuka et al.

Neuromagnetic responses to chords are modified by preceding musical scale 